See discussions, stats, and author profiles for this publication at: https://www.researchgate.net/publication/323226935

\title{
Performance Analysis of Analog IF Over Fiber Fronthaul Link With 4G and 5G Coexistence
}

Article in Journal of Optical Communications and Networking · March 2018

DOI: $10.1364 /$ JOCN.10.000174

CITATIONS

29

8 authors, including:

Amol Delmade

Dublin City University

16 PUBLICATIONS 51 CITATIONS

SEE PROFILE

Nicola Marchetti

Trinity College Dublin

201 PUBLICATIONS 1,582 CITATIONS

SEE PROFILE

Some of the authors of this publication are also working on these related projects:

Project Phase Control in Coherent Beam Combining View project

Project High power lasers View project
READS

712

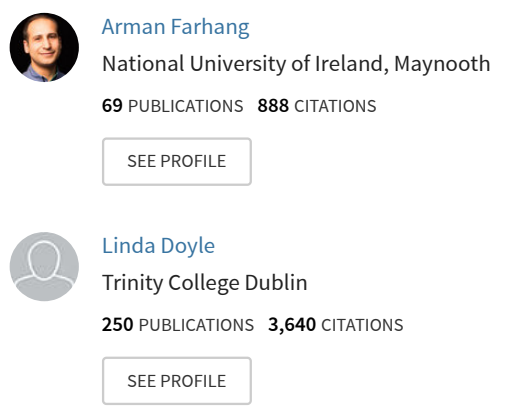




\title{
Performance Analysis of Analog IF over Fiber Fronthaul link with 4G \& 5G Co-existence
}

\author{
Amol Delmade, Colm Browning, Arman Farhang, Nicola Marchetti, Linda E. Doyle, \\ David Koilpillai, Liam P. Barry, and Deepa Venkitesh
}

\begin{abstract}
Fifth generation (5G) mobile communications will require a dense deployment of small cell antenna sites and higher channel bandwidth, in conjunction with a cloud radio access network (C-RAN) architecture. This necessitates low latency and high capacity architecture in addition to energy and cost efficient fronthaul links. An efficient way of achieving such connectivity is to make use of optical fiber based infrastructure where multiple wireless services may be distributed over the same fiber to remote radio head (RRH) sites. In this work, we demonstrate the spectral containment of 4G long term evolution (LTE) signal and 5G candidate waveforms - generalized frequency division multiplexing (GFDM) and universally filtered orthogonal frequency division multiplexing (UF-OFDM), through a directly modulated link. 75 bands of LTE and 10 bands of 5G waveforms are successfully transmitted over $25 \mathrm{~km}$ analog intermediate frequency signal over fiber (AIFoF) link through our setup, limited only by the bandwidth of the laser. For the first time, we demonstrate the fronthaul network for providing simultaneous 4G \& 5 G services by propagating LTE signals in coexistence with UF-OFDM.
\end{abstract}

Index Terms-4G; Long Term Evolution; 5G; Universally Filtered Orthogonal Frequency Division Multiplexing; Generalized Frequency Division Multiplexing; Analog IF over Fiber.

\section{INTRODUCTION}

$\mathbf{T}$ HE appetite for broadband and successful deployment of a wide range of applications has fueled the continual upgrade of mobile cellular networks. With the increasing demand for machine-to-machine communication, the mobile networks must scale appropriately in order to provide higher speeds with increased flexibility to a variety of users [1]. As the long term evolution (LTE) system embodying 4G has now been deployed and is reaching maturity, cellular industry and researchers are targeting standardization of 5G mobile communication protocol. The engineering requirement of meeting the edge data rate of $100 \mathrm{Mbps}$ (100 times that of $4 \mathrm{G}$ ) for $95 \%$ of users will require the ultra-dense deployment of small cell antenna sites, increased bandwidth by moving toward millimeter wave (MMW) frequencies and advances in MIMO technology in conjunction with a centralized radio access network (C-RAN) architecture for better coordination among cells for minimizing the interference [2].

In C-RAN architecture, the functionalities of the traditional base station are split between the baseband unit (BBU) and remote radio head $(\mathrm{RRH})$ unit. Centralized baseband units $(\mathrm{C}$ $\mathrm{BBU})$ perform the baseband processing of multiple BBUs at a

Amol Delmade, David Koilpillai and Deepa Venkitesh (e-mail: deepa@ee.iitm.ac.in) are with the Department of Electrical Engineering, Indian Institute of Technology, Madras 600036, India.

Colm Browning, and Liam P. Barry are with School of Electronics Engineering, Dublin City University, Glasnevin, Dublin 9, Ireland.

Arman Farhang, Nicola Marchetti and Linda E. Doyle are with the Connect Research Center, Trinity College Dublin, Dublin 2, Ireland. common location, while simpler RRH units perform the radio processing with reduced energy consumption at the antenna site. This requires $\mathrm{C}-\mathrm{BBU}$ and $\mathrm{RRH}$ units to be connected through a high speed, low latency and accurately synchronized network, referred to as the fronthaul [3]. These requirements make optical fiber an obvious choice for connecting CBBU and RRH sites. Signals can be transmitted through the fronthaul network in analog or digital domain at baseband or passband with respect to an optical carrier, resulting in different fronthaul schemes [3].

Digitized baseband signal over fiber (DBBoF) based common public radio interface (CPRI) mobile fronthaul scheme requires $32 \mathrm{~Gb} / \mathrm{s}$ optical line rate for serving a four sector $8 \times 8 \mathrm{MIMO}$ antenna site for $20 \mathrm{MHz}$ bandwidth wireless channel [4]. CPRI fronthauling architecture becomes more inefficient and complex for providing multiple services through the same fiber. Analog-domain radio frequency signal over fiber (ARFoF) schemes increase the bandwidth efficiency and reduce the latency by avoiding the use of expensive $\mathrm{A} / \mathrm{D}$ and $\mathrm{D} / \mathrm{A}$ converters at the RRH for the digitization process, as required by CPRI [3]. Traditional ARFoF however requires optical components that work at bandwidths of at least the carrier frequencies. Transmitting analog-domain radio signals at intermediate frequencies (IF) over fiber (AIFoF) provides more flexibility and uses lower bandwidth optical components compared to radio/MMW frequency signal over fiber technique required for $5 \mathrm{G}$ signals fronthauling [5]. The schematic of a basic AIFoF scheme for a downlink scenario is shown in Fig. 1, where the baseband signal is converted to IF frequencies in the $\mathrm{C}-\mathrm{BBU}$ before transmission through fiber, which is further up-converted to the desired RF signal at the RRH. We had performed a detailed comparison of the different fronthauling schemes to prove the efficacy of the AIFoF scheme in [6].

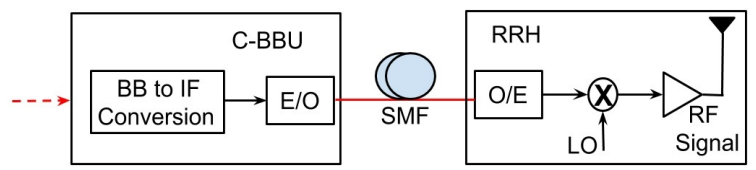

Fig. 1: Block diagram of an AIFoF system for downlink.

Multicarrier modulation based orthogonal frequency division multiplexing (OFDM) waveforms have been adopted in the majority of wireless standards, such as 4G LTE [7] and wireless local area network (WLAN) technology [8], because of its inherent advantages in fading environments. Digital implementations using fast Fourier transform (FFT) and its inverse give higher out-of-band (OOB) emission. High peak- 
to-average power ratio (PAPR) and difficulty in maintaining orthogonality - especially for uplink transmission - also contributes to limitations of OFDM as a contending $5 \mathrm{G}$ waveform [9]. Generalized frequency division multiplexing (GFDM) and universally filtered orthogonal frequency division multiplexing (UF-OFDM) have emerged as potential candidate waveforms for $5 \mathrm{G}$ mobile communication [9]. GFDM performs a per-subcarrier filtering, while UF-OFDM filters at the resource block level to reduce the OOB emission compared to OFDM, as described in more detail in section II.

In addition to these high bandwidth $5 \mathrm{G}$ signals, low bandwidth legacy services such as GSM, CDMA and LTE are also expected to be supported in the future network. Thus it is imperative to design the optical fronthaul network such that the performance of both high and low bandwidth services are guaranteed in situations where they co-exist.

Fronthauling of 4G LTE signals using AIFoF technique has previously been demonstrated in [10] for $20 \mathrm{MHz}$ signals, while in [11] a comparison of gain for direct and external modulation links is done for 4 bands of $100 \mathrm{MHz}$. A complex fronthauling network architecture for the $5 \mathrm{G}$ candidate waveform filter-bank multi-carrier (FBMC) using external modulation is shown in [12]. A combined fronthaul link for $200 \mathrm{MHz}$ OFDM and $20 \mathrm{MHz}$ LTE signals is demonstrated in [13], but it uses different wavelengths to modulate different services requiring a photo-detector (PD) with $70 \mathrm{GHz}$ bandwidth at the RRH site. Advantages offered by UF-OFDM over GFDM and OFDM, in terms of performance for reduced guard-bands between the wireless signal bands in convergence with pulse amplitude modulation-4 (PAM) based passive optical network has been studied in our previous work [14].

In this paper, we demonstrate the transmission of different wireless services with variable baseband sample rate and OOB emissions over the same AIFoF fronthaul link that feeds data to a heterogeneous RRH site. We analyze the effect on the performance of $20 \mathrm{MHz}$ LTE signals in coexistence with $201 \mathrm{MHz}$ [15] 5G candidate waveform - UF-OFDM - through a low cost directly modulated laser based AIFoF fronthaul link. In order to analyze system performance we first demonstrate the spectral containment of $20 \mathrm{MHz}$ LTE signals in the AIFoF system. Then similar transmission is carrier out using $201 \mathrm{MHz}$ bands of $5 \mathrm{G}$ candidate waveforms - UF-OFDM and GFDM. Transmission with OFDM in place of the $5 \mathrm{G}$ waveforms is also demonstrated for performance comparisons.

The paper is organized as follows: Section II presents details of waveforms - OFDM, GFDM and UF-OFDM along with details of LTE standard, Section III describes the experimental setup and details of the parameters used for generation of various modulating signals. In Section IV, the experimental results are discussed in detail and the results are summarized in Section V.

\section{Details of WaVeforms}

\section{A. Orthogonal Frequency Division Multiplexing}

OFDM achieves high speed data transmission by dividing the serial data into multiple low speed parallel data channels modulated on different frequencies resulting in longer symbol periods. FFT operation on the symbols can simultaneously modulate and multiplex data as shown in Fig. 2(a). IFFT basically multiplies the incoming parallel QAM data with orthogonal frequency sinusoids and then adds to get one OFDM symbol [9]. Each IFFT operation gives one OFDM symbol; a number of such symbols are transmitted serially. The period of an OFDM symbol after an $N$-point IFFT operation is $N$ times longer than the input QAM data. Sub-carriers which are aligned at the transmitter, after propagation through a dispersive channel, appear at the receiver with different delays, leading to an inter-symbol interference (ISI).

After the IFFT operation, $N_{C P}$ samples from the end of symbol are appended at the beginning, generating a cyclic

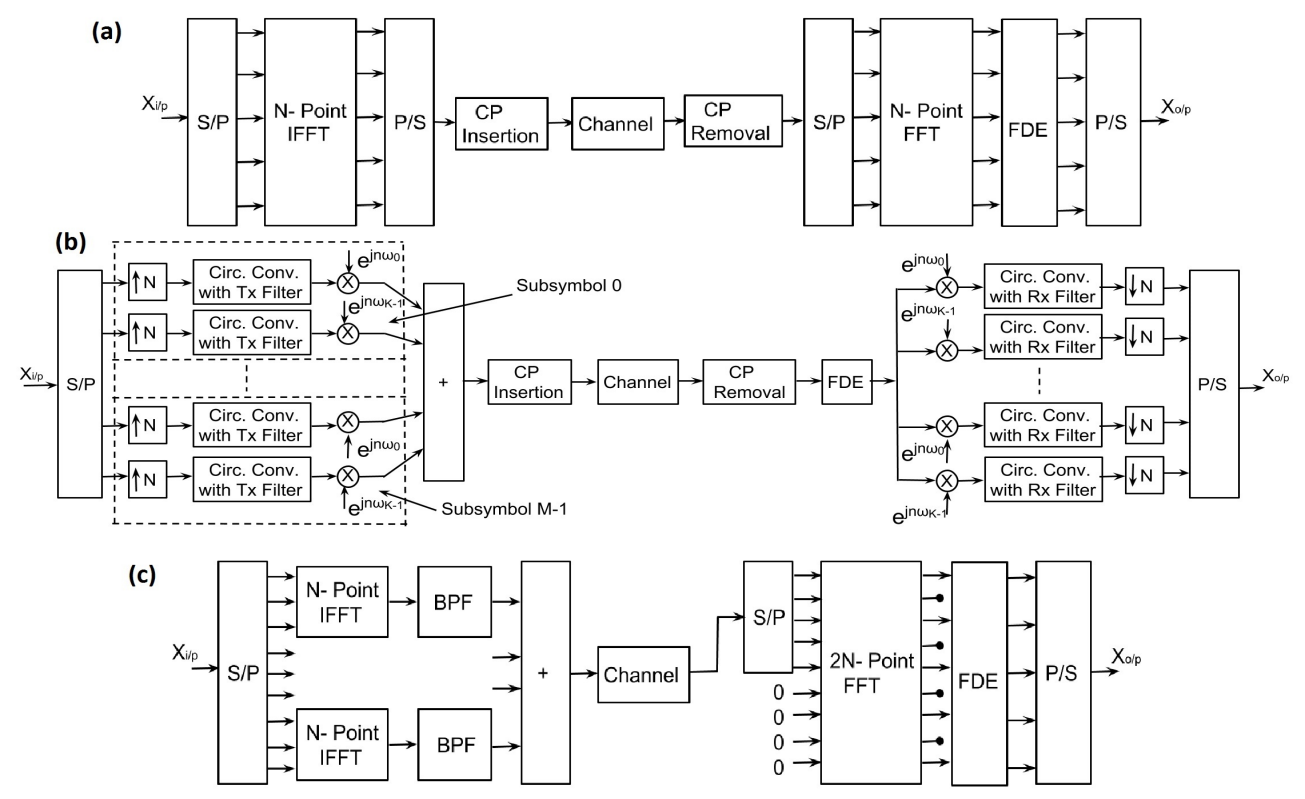

Fig. 2: Baseband structure of (a) OFDM, (b) GFDM and (c) UF-OFDM. 
prefix (CP) in order to avoid ISI due to fading [9]. It also converts the linear convolution of the transmit signal with the channel impulse response (CIR) into a circular convolution - requiring a single-tap frequency domain equalizer (FDE) at the receiver. $\mathrm{CP}$ leads to a bandwidth efficiency loss of $\frac{N_{C P}}{N}$. Moreover, OFDM suffers from a large amount of OOB emission, as evident from sinc pulse shape of subcarrier in the frequency domain, thus requiring large guard bands between multiple OFDM signals.

\section{B. Generalized Frequency Division Multiplexing}

GFDM applies circular pulse filtering on each subcarrier to minimize the overlapping with adjacent subcarriers. It increases the tolerance to timing synchronization errors and carrier frequency offset [16]. The baseband structure of a GFDM transceiver is shown in Fig. 2(b). A block of input QAM symbols is split into $N$ parallel sub-streams to fill $K$ subcarriers frequencies and $M$ time sub-symbols [17].

The data of each stream is up-sampled by a factor of $N$, which essentially multiplies data with an impulse $\delta[n-m K]$ and introduces a time shift of $m K$ samples, which moves each data symbol to the correct sub-symbol position in the GFDM block [17]. Circular convolution is done with the transmitter prototype filter to remove the replicas generated as a result of upsampling. All the subcarriers are superposed after shifting to their corresponding center frequencies. $\mathrm{CP}$ longer than the channel impulse response is inserted. An interesting property of GFDM is that it requires only one CP for $M$ sub-symbols, thus reducing the signal overhead.

After a GFDM block is received, CP is removed and reverse operations are implemented to retrieve the data. Equalization is performed to compensate for channel distortions, after downsampling, similar to that in OFDM. The rectangular truncated number of pure tones transmitted by GFDM gives high OOB emission, similar to OFDM. More details about GFDM and its relation with OFDM can be found in [9]. Individual subsymbol detection is impossible for GFDM, since only one CP is used for $M$ sub-symbols, thus increasing the latency. For a low complexity implementation of GFDM, we deploy the modem structure that is proposed in [18] while using the zeroforcing $(\mathrm{ZF})$ equalizer. It is known that GFDM suffers from some performance penalty compared with OFDM which is due to the non-orthogonality of its subcarriers [17], [18].

\section{Universally Filtered Orthogonal Frequency Division Mul- tiplexing}

UF-OFDM performs linear filtering on sub-bands of subcarriers, thus reducing the filter length as shown in Fig. 2(c) [9]. Input QAM symbols are mapped to the allocated sub-bands of subcarriers while setting the subcarriers at the position of the remaining sub-bands zero. Bandpass filtering is done after taking the $N$-point IFFT on each sub-band data. The modulated version of a Dolph-Chebyshev bandpass filter is used to tackle the OOB emissions as well as the in-band leakage problems of OFDM [19]. The UF-OFDM transmit signal is formed after superposition of the bandpass filtered sub-band signals. Overhead in UF-OFDM is no more than that required for the $\mathrm{CP}$ of an equivalent OFDM signal, enhancing the spectral containment compared to OFDM [19].

$N-L$ zeros are padded to the received symbol after propagation through the channel, where $L$ is the filter length used at transmitter. $2 N$-point FFT operation is performed to demodulate the received UF-OFDM signal. A single-tap equalization is done on the odd output bins, neglecting the even outputs of the FFT block, given perfect knowledge of the channel response [9]. To reduce the computations, an $N$ point FFT operation can be performed along with the FDE at the receiver by performing time aliasing before feeding the signal to FFT block [9]. Details of waveform parameters used and corresponding spectra are discussed in section III.

\section{EXPERIMENTAL SETUP}

\section{A. Direct Modulation for Fronthaul}

Optical modulation is possible either by directly modulating the laser current or by external modulation. External modulation, using a Mach Zehnder modulator (MZM), introduces insertion losses and polarization sensitivity, as well as increasing transmitter footprint and cost, compared to a direct modulation scheme. Direct modulation does not require strict bias control or a polarization controller (PC), and suits best for wide deployment in fronthaul application due to its low cost and simple implementation. However, directly modulated lasers exhibit a resonant peak in modulation response, arising from the nonlinear interactions between photons and carriers in the laser cavity, beyond which the frequency response of the laser decreases [20]. Modulating a signal in the nonlinear region degrades the link performance, so it is important to know the

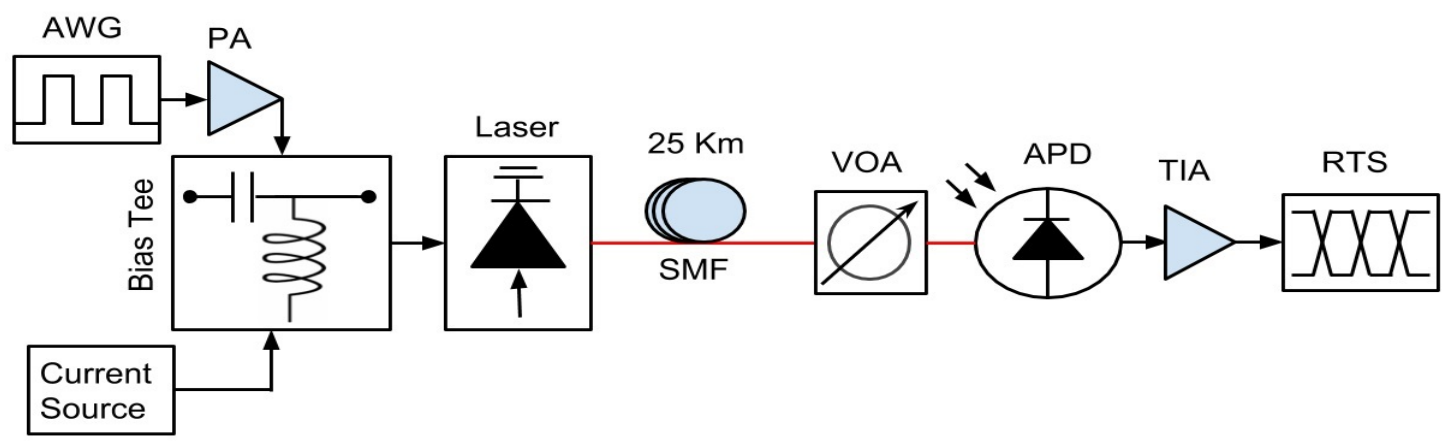

Fig. 3: Experimental setup to demonstrate the optical fronthauling of the $4 \mathrm{G}$ and $5 \mathrm{G}$ waveforms through intensity modulation direct detection system. 
modulation characteristics of the laser before deciding on an appropriate intermediate frequency.

\section{B. Setup}

Fig. 3 shows the schematic of the experimental setup. Raw data corresponding to the intermediate frequency modulated 4G and 5G signals are generated offline and loaded on an arbitrary waveform generator (AWG) operating at $10 \mathrm{GS} / \mathrm{s}$. Light from a discrete mode laser diode [20], is directly modulated by the AWG output signal after RF amplification. The frequency response of this laser is measured using a vector network analyzer (VNA) and shown in Fig. 4, indicating a resonant peak at $2.25 \mathrm{GHz}$ for a bias current of $50 \mathrm{~mA}$. The input average power to the $25 \mathrm{~km}$ of single mode fiber (SMF) is maintained at $\sim 1 \mathrm{dBm}$. A variable optical attenuator (VOA) is used to control the input optical power to the avalanche photodiode (APD - bandwidth of $10 \mathrm{GHz}$ ) which exhibits saturation close to $-10 \mathrm{dBm}$. Maximum received optical power at APD is limited to $-13 \mathrm{dBm}$. The output of trans-impedance amplifier (TIA) is sampled at $25 \mathrm{GS} / \mathrm{s}$ by a real time oscilloscope (RTS). Error vector magnitude (EVM) analysis is done offline after equalizing the digitally filtered and down converted signal.

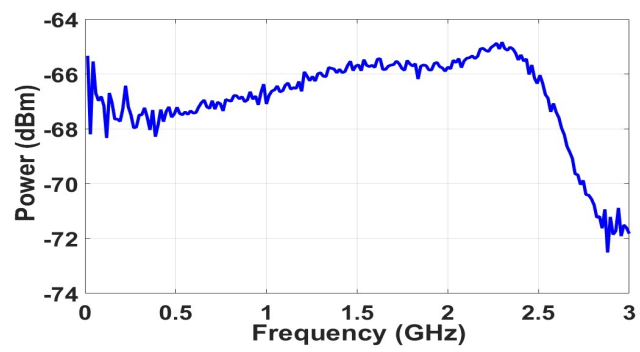

Fig. 4: Frequency response of the direct modulation laser, measured using a VNA; bias current $=50 \mathrm{~mA}$.

\section{Signal Generation}

Multiple bands of LTE signals are generated in Matlab, with 1200 subcarriers out of 2048 modulated using 16 QAM data and remaining subcarriers packed with zero. Subcarrier spacing of $15.25 \mathrm{KHz}$ results in a raw data rate of $\sim 73$ $\mathrm{Mb} / \mathrm{s}$ for $20 \mathrm{MHz}$ band of LTE signal. Similarly multiple bands of 5G waveforms GFDM, UF-OFDM and OFDM are generated separately, with baseband bandwidth of $201 \mathrm{MHz}$ with different overheads.

\begin{tabular}{|c|c|c|c|}
\hline Parameters & OFDM & GFDM & UF-OFDM \\
\hline Sampling Freq (Ms/s) & 625 & 625 & 625 \\
\hline IFFT Size & 1024 & 1024 & 1024 \\
\hline Subcarrier Spacing (KHz) & 610.35 & 610.35 & 610.35 \\
\hline Data Subcarriers & 330 & 330 & 330 \\
\hline Number of sub bands & n/a & n/a & 13 \\
\hline Overlapping Factor & n/a & 7 & n/a \\
\hline Filter Type & n/a & PHYDYAS & $\begin{array}{l}\text { Dolph Cheby } \\
\text { chev FIR }\end{array}$ \\
\hline Filter tap length (Samples) & n/a & 13 & 74 \\
\hline Cyclic Prefix (\%) & 6.25 & 6.25 & n/a \\
\hline Raw data rate & $0.81(\mathrm{~Gb} / \mathrm{s})$ & $0.81(\mathrm{~Gb} / \mathrm{s})$ & $0.81(\mathrm{~Gb} / \mathrm{s})$ \\
\hline
\end{tabular}

TABLE I: $5 \mathrm{G}$ waveforms parameters

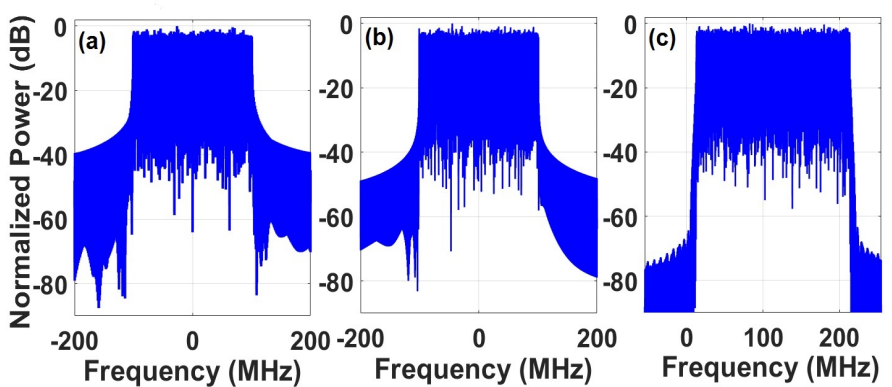

Fig. 5: Baseband spectra of single bands of (a) OFDM, (b) GFDM and (c) UF-OFDM for a bandwidth of $201 \mathrm{MHz}$ in each case.

330 subcarriers out of 1024 are modulated with 16 QAM data, while remaining subcarriers packed with zero, at a subcarrier spacing of $610.35 \mathrm{KHz}$ for all $5 \mathrm{G}$ waveforms resulting in a raw data rate of $0.81 \mathrm{~Gb} / \mathrm{s}$ per band. In all cases the multicarrier waveforms are hard clipped to $80 \%$ of their original maximum resulting in reduced PAPR, which is between 12$14 \mathrm{~dB}$. Specific properties of each $5 \mathrm{G}$ waveform can be found in Table I. The baseband spectra of each of these signals are shown in Fig. 5, where the OOB emission characteristic of each waveform can be clearly observed. The effect of sub-band linear filtering used with UF-OFDM (Fig. 5(c)) is evident since the OOB emission is highly reduced in this case compared to both GFDM (Fig. 5(b)) and OFDM (Fig. 5(a)).

Both $4 \mathrm{G}$ and $5 \mathrm{G}$ waveform bands are added together digitally after upsampling and modulated onto different intermediate frequencies. The intermediate carrier frequencies for these bands are chosen to be located in the linear modulation region of the laser, which is below $2.25 \mathrm{GHz}$ for $50 \mathrm{~mA}$ bias current. We biased the laser at $67 \mathrm{~mA}$, which shifts the relaxation oscillation peak to a frequency [20] higher than shown in Fig. 4. A signal swing of $70 \mathrm{~mA}$ is maintained at the RF input of the laser diode. At the receiver, the signals are resampled and a $12^{t h}$ order Gaussian filter is used to extract each band for processing.

\section{RESULTS AND DISCUSSION}

We first evaluate the performance of multi-band LTE, UFOFDM and GFDM waveforms through the AIFoF link, when transmitted independently. We further compare this performance in the scenario where the multiple waveforms co-exist.

\section{A. AIFoF for LTE}

RF carrier frequencies for LTE signal range from 700 $\mathrm{MHz}$ to $3600 \mathrm{MHz}$, so signals can be transmitted through the fronthaul network without IF conversion, with appropriate choice of lasers and detectors. For multi-sector operation, the wireless signals corresponding to different directions could have similar carrier frequencies, but needs to be transmitted independently through the fronthaul link. This necessitates different IF frequencies for each sector. The passive optical fronthaul link does not add any signal to noise ratio (SNR) degradation except for the shot noise and the thermal noise at the receiver. Dispersion induced RF fading can be ignored for IF frequencies for fiber lengths of $<50 \mathrm{~km}$, and the 
contributions due to third order inter-modulation products are found to be minimal in our receiver. For the transmission of a single $20 \mathrm{MHz}$ LTE signal centered at $1.5 \mathrm{GHz}$ of IF carrier, the EVM is observed to be well below the forward error correction (FEC) limit (12.5\%) for 16 QAM data modulation.

Typically, the chosen guard-band in multi-bands transmission systems are selected to minimize the channel interference. A spectral guard-band of $2 \mathrm{MHz}$ (10\% of the signal bandwidth [21]) is chosen between the LTE bands. Signals are added on either side of band centered at $1.5 \mathrm{GHz}$ with IF carriers $21 \mathrm{MHz}$ apart as shown in the modulating signal spectra of 8 bands LTE in Fig. 6.

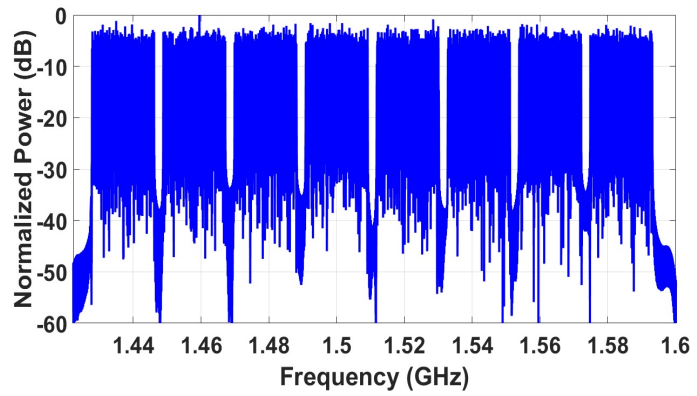

Fig. 6: Modulating signal spectra for eight bands of LTE.

The EVM performance of each of the bands is shown in Fig. 7 as a function of received optical power. All eight signal bands are found to exhibit performance below the FEC limit for a received optical power $\left(P_{r x}\right)$ of $-22 \mathrm{dBm}$ or higher. This eight band transmission link offers an optical power budget of $18 \mathrm{~dB}$ considering $5 \mathrm{~dB}$ loss in the $25 \mathrm{~km}$ fiber and a transmitted power $\left(P_{t x}\right)$ of $\sim 1 \mathrm{dBm}$.

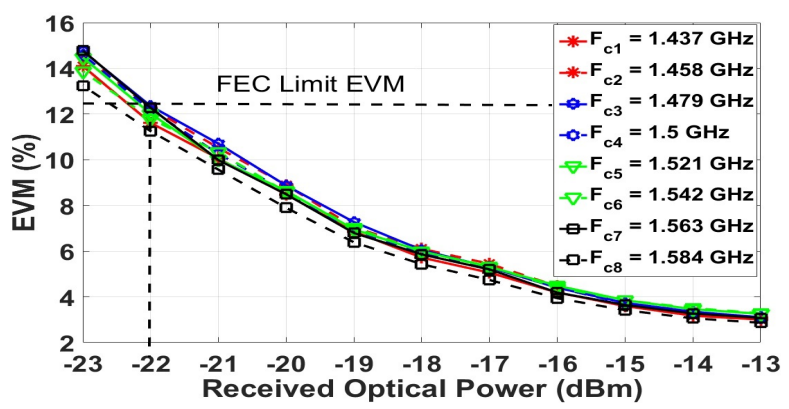

Fig. 7: EVM performance for eight bands of LTE for 16 QAM AIFoF transmission.

We now repeat the experiment with 20 and 75 bands of LTE. The EVM performance for the 20 and 75 bands as a function of carrier frequency at the received optical power of $-13 \mathrm{dBm}$ is shown in blue and red, respectively, in Fig. 8. The blue curve shows that the EVM values for all the 20 bands are almost same and is less than $4.15 \%$. The red curve indicates that EVM achieved is $\sim 7.25 \%$, which is well within the FEC limits, thus proving that AIFoF link does support 75 bands of LTE - corresponding to a raw data rate of $5.475 \mathrm{~Gb} / \mathrm{s}$ with an occupied bandwidth of $\sim 1.6 \mathrm{GHz}$. The difference between the EVMs across the bands is negligibly small $(<1 \%)$. The overall performance is however degraded with the addition of more signal bands since the total power - maintained constant in both the experiments - is shared between more number of

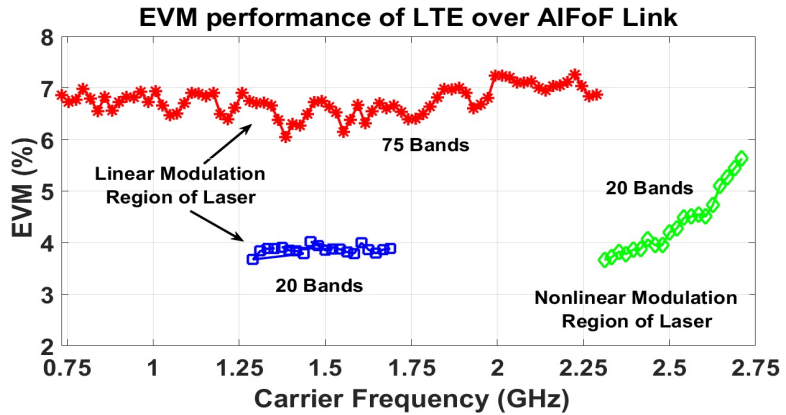

Fig. 8: EVM performance for $20 \& 75$ bands of LTE modulated with different IF frequencies.

signals in the 75 band transmission case. Maximum received optical power is limited to $-13 \mathrm{dBm}$ in this case and lower EVMs could be obtained with the higher received power.

In order to transmit the LTE signals with higher IF carrier frequencies, multiple bands were added on either side of the band centered at $2.5 \mathrm{GHz}$ frequency, and transmitted through the fronthaul link. Like in the previous case, the total power launched into the fiber is maintained as a constant $(1 \mathrm{dBm})$. The EVM performance for a received power of $-13 \mathrm{dBm}$ for 20 bands in this case is shown in green in Fig. 8. It is evident from the green curve that the performance of higher frequency bands significantly degrade. This degradation in performance among the bands can be directly correlated with the laser response, shown in Fig. 4. Nevertheless, overall performance for the case with 20 bands is better compared to that with 75 bands because of the larger power available per band. Thus, when analog IFoF scheme is used with direct modulation, there is a limit on the number of bands that can be transmitted through the system, and this needs to be evaluated based on the optical power levels and bandwidth of the laser used. We now proceed to evaluate the performance of $5 \mathrm{G}$ waveforms.

\section{B. AIFoF for $5 G$}

Wireless carrier frequency bands ranging from $6 \mathrm{GHz}$ to $100 \mathrm{GHz}$ are proposed for 5G [22]. We have implemented analog intermediate frequency over fiber (AIFoF) fronthauling scheme, which uses carriers frequencies smaller than the frequencies of the proposed $5 \mathrm{G}$ systems. Signals are frequency shifted to the desired wireless carrier frequency band after detection at the RRH site as shown in the Fig. 1, by using the RF mixer and local oscillator. The range of IF carrier frequencies that can be used for transporting signals between $\mathrm{C}-\mathrm{BBU}$ and RRH sites depends on the capabilities of the components used for the fronthaul link. The bandwidth in our experimental implementation is limited by that of the direct modulation laser; thus, the range of IF carrier frequencies is limited to $2.4 \mathrm{GHz}$.

To evaluate the performance of $5 \mathrm{G}$ candidate waveforms, a single band of $201 \mathrm{MHz}$ bandwidth UF-OFDM and GFDM baseband signal is mixed with a $1.5 \mathrm{GHz}$ IF carrier and transmitted separately through the AIFoF fronthaul link. Fig. 9 shows the EVM vs received power performance for UFOFDM and GFDM compared with OFDM. UF-OFDM and OFDM exhibits similar performance, as UF-OFDM's lower OOB emission property does not have any bearing on system 
performance in this single band case. For the same operating

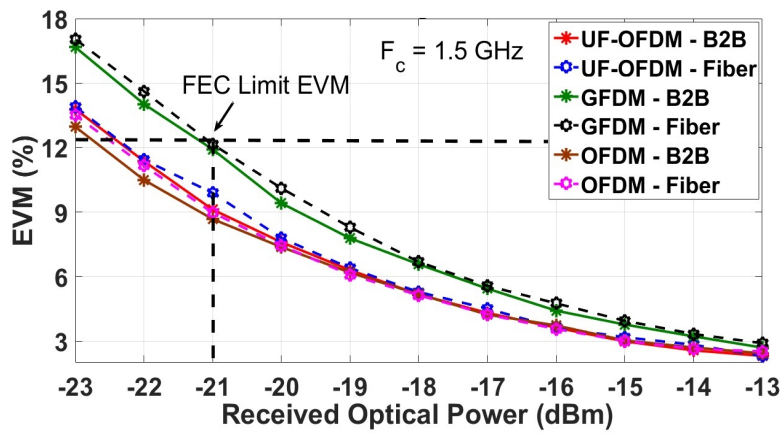

Fig. 9: EVM performance for a single band of UF-OFDM, GFDM and OFDM.

conditions, GFDM exhibits a larger EVM penalty compared to UF-OFDM and OFDM for the same power and this is attributed to noise enhancement caused by the non-orthogonality of the GFDM subcarriers, as outlined in section II.B. All three of the waveforms can be successfully demodulated for $P_{r x} \geq$ $-21 \mathrm{dBm}$. This link offers an optical power budget of $17 \mathrm{~dB}$ considering $5 \mathrm{~dB}$ loss in the $25 \mathrm{~km}$ fiber for $P_{t x} \sim 1 \mathrm{dBm}$. EVM at maximum received power of $-13 \mathrm{dBm}$ is $\sim 3 \%$ for all three signals, which is also observed for the case of $20 \mathrm{MHz}$ LTE as seen from 8 band transmission results of Fig. 7. Thus, increasing the bandwidth from $20 \mathrm{MHz}$ to $201 \mathrm{MHz}$ does not degrade the performance of the AIFoF link for higher optical power level.

As in the case of LTE, we now transmit multiple bands of these $5 \mathrm{G}$ waveforms through the fronthaul link and evaluate their performance. Spectral guard-band of $20 \mathrm{MHz}$ is chosen between 5G signal bands for multi-band transmission of all three waveforms. Fig. 10 shows the EVM of the three waveforms in this multi-band scenario (with ten bands), as a function of the carrier frequency (similar to Fig. 8 for LTE), at two received optical power levels of $-13 \mathrm{dBm}$ and -17 $\mathrm{dBm}$. The figure also indicates the EVM for a single band transmission scenario. Comparing with Fig. 9, the performance

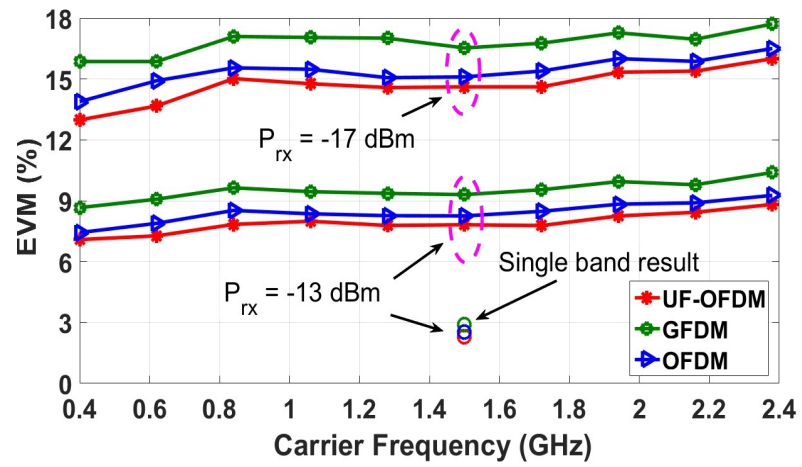

Fig. 10: EVM for ten bands of OFDM, GFDM \& UF-OFDM after transmission through AIFoF fronthaul link.

has degraded with addition of signal bands as all the ten bands of the three waveforms exhibit EVM between 7 to $10 \%$ at $P_{r x}$ of $-13 \mathrm{dBm}$.

This degradation is primarily attributed to the power sharing in multi-band case, since the total power level into the fiber is maintained constant in the single and multi-band experiment. The results indicate a slight degradation in system performance as IF carrier frequencies approach the nonlinear operation of the laser - beyond $2.25 \mathrm{GHz}$ - where EVM values increases by $2 \%$, as compared to lower frequency bands. Results show the further increase in EVM as the received optical power is reduced, thus degrading SNR. For $P_{r x}>-16 \mathrm{dBm}$, all the ten bands of three waveforms show EVM $<12.5 \%$, indicating a successful transmission over fronthaul link, where a raw data rate of $8.1 \mathrm{~Gb} / \mathrm{s}$ is achieved. It can be observed that GFDM performs worse than both UF-OFDM and OFDM due to the non-orthogonality of the GFDM subcarriers. UF-OFDM and OFDM exhibit similar performance in the single band case (results shown in Fig. 9), as the smaller out-of-band (OOB) emission property of UF-OFDM does not have any bearing on system performance. However, in the ten-band transmission scenario (results shown in Fig. 10), lower OOB emission of UF-OFDM results in a better performance compared to OFDM as indicated by the EVM curves. Since the performance of UFOFDM is more tolerant to inter-band interference [14], a large number of bands may be supported.

A fair comparison of several $5 \mathrm{G}$ candidate multicarrier waveform has been conducted in [9], [16], [23], [24]. Some important aspects of UF-OFDM/UFMC waveform highlighted in [23] are : enhanced performance in multi-user scenario, comparable SE with OFDM and backward compatibility with well-known OFDM algorithms. Some issues of GFDM such as increased transceiver complexity, adaptability to MIMO transmission systems and short duration packet adaptation needs to be addressed [23]. However, the advantages of GFDM such as tolerance to synchronization errors [16] as well as increased spectral efficiency, compared to UF-OFDM, must be considered while designing waveforms for future communication system. Aspects related to synchronization of these waveforms are thoroughly investigated in [16] and [24]. The performance differences between the FDM waveforms are only due to those arising in the electrical domain since the penalty introduced by the optical AIFoF fronthauling link is agnostic to these waveforms at the bandwidths considered.

Performance of time varying bit rate signals, as envisioned by $5 \mathrm{G}$, will not change over the optical fronthaul link operated with AIFoF scheme as long as their bandwidth and power characteristics are maintained within the system limits. We expect that, as our system is largely agnostic to changes in the underlying waveform conditions, the results presented in this work are valuable within this context.

Also future $5 \mathrm{G}$ systems should be capable of reconfiguring within reasonable times, which is expected to lead into the maximum end-to-end latencies of $1 \mathrm{~ms}$. AIFoF fronthauling is not expected to add any additional latencies apart from the fiber transmission delay. In our experiments $\mathrm{C}-\mathrm{BBU}$ and RRH units are separated by $25 \mathrm{~km}$ of single mode fiber (SMF), which adds a round trip latency of about $250 \mu \mathrm{s}$ $\left(2 \times(\right.$ distance/velocity $\left.)=2 \times\left(\left(25 \times 10^{3}\right) /\left(2 \times 10^{8}\right)\right)\right)$ into the system. This distance for our experiments was chosen by considering LTE system latency, but is ultimately decided by the latency requirements of $5 \mathrm{G}$. We now proceed to analyze the performance of multiple bands of signals carrying different 
bit-rate.

\section{AIFoF for coexistence of LTE \& UF-OFDM}

We now demonstrate the co-existence of LTE and UFOFDM waveforms. We take an example where a fronthaul network is designed for serving the most commonly used 3 sector RRH site consisting of $2 \times 2$ MIMO scenario (6 signals) for $201 \mathrm{MHz}$ UF-OFDM (4.86 Gb/s) and 4x4 MIMO scenario (12 signals) for $20 \mathrm{MHz}$ LTE (876 Mb/s). IF carrier

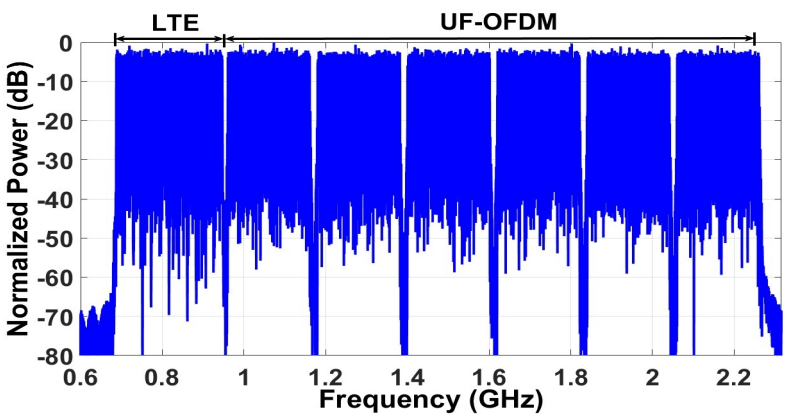

Fig. 11: Spectrum of LTE and UF-OFDM signals in coexistence AIFoF fronthaul network.

frequencies from $696 \mathrm{MHz}$ to $938 \mathrm{MHz}$ and $1.06 \mathrm{GHz}$ to 2.16 $\mathrm{GHz}$ are used for transmitting LTE and UF-OFDM signals with $2 \mathrm{MHz}$ and $20 \mathrm{MHz}$ inter band spacing respectively as shown in spectra of Fig. 11. Signals can be freely assigned independent of the bandwidth by maintaining sufficient guard bands with both neighboring bands.

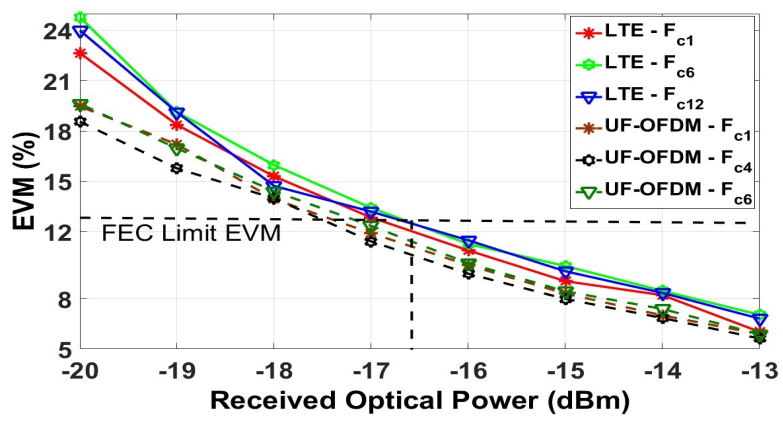

Fig. 12: EVM of LTE and UF-OFDM signals in coexistence AIFoF fronthaul network.

Fig. 12 shows the EVM performance for LTE and UFOFDM bands in the above mentioned coexistence scenario. UF-OFDM signal bands can be successfully demodulated for $P_{r x} \geq-16 \mathrm{dBm}$ - indicating similar sensitivity to that observed where 10 bands of $5 \mathrm{G}$ were transmitted (Fig. 10). The performance of LTE degrades in this case as 7\% EVM is observed at $P_{r x}=-13 \mathrm{dBm}$, which is also observed in the case of the transmissions of 75 LTE bands as shown in Fig. 8. It is to be noted that the total bandwidth occupied by modulating signal in coexistence case is same as occupied by the 75 bands of LTE. Hence performance degradation can be attributed to the lower power sharing to LTE signals in coexistence scenario. Also OFDM based LTE bands shows a worse performance, compared to UF-OFDM, which can be attributed to the increased inter-band interference.
Performance of this fronthaul transmission link is limited by the bandwidth of laser used for this experiment. Using a laser with extended direct modulation bandwidth will allow for increased throughput in AIFoF links. Also maximum received optical power is limited to $-13 \mathrm{dBm}$ in this case because of APD saturation. Received power could be increased up to -4 $\mathrm{dBm}$ considering the transmitted optical power of $1 \mathrm{dBm}$ and fiber loss of $5 \mathrm{~dB}$.

\section{CONCLUSION}

For the first time, direct modulation based spectral containment of 4G LTE and 5G candidate waveforms, GFDM and UFOFDM, has been experimentally demonstrated using AIFoF fronthauling scheme. Seventy-five and ten bands of $20 \mathrm{MHz}$ LTE and $201 \mathrm{MHz} 5 \mathrm{G}$ candidate waveforms are transmitted separately over $25 \mathrm{~km}$ fronthaul network, respectively, showing the potential of link for providing services to multiple RRH sites. Effect on the performance of LTE in coexistence with UF-OFDM for transmission through same fronthaul link is demonstrated. Results shows that EVM of LTE and UF-OFDM bands are well below the FEC limit, showing successful delivery of different wireless services with different baseband sample rates through same fronthaul network. Performance of these links can be further improved by using higher modulation bandwidth lasers or external modulation and higher saturation power APD's.

\section{ACKNOWLEDGMENT}

This work has been jointly supported through the EU UPPI project (PIRSES-GA-2012-318941), SFI US-Ireland project (15/US-C2C/I3132), CONNECT (13/RC/2077), and IPIC (12/RC/2276) research grants.

\section{REFERENCES}

[1] G. Wunder, P. Jung, M. Kasparick, T. Wild, F. Schaich, Y. Chen, S. T. Brink, I. Gaspar, N. Michailow, A. Festag, L. Mendes, N. Cassiau, D. Ktenas, M. Dryjanski, S. Pietrzyk, B. Eged, P. Vago, and F. Wiedmann, "5G NOW : Non-orthogonal, Asynchronous waveforms for future mobile applications," IEEE Communications Magazine, vol. 52, no. 2, pp. $97-$ 105, February 2014.

[2] J. G. Andrews, S. Buzzi, W. Choi, S. V. Hanly, A. Lozano, A. C. K Soong, and J. C. Zhang, "What Will 5G Be?," IEEE Journal on Selected Areas in Communications, vol. 32, no. 6, pp. 1065-1082, June 2014.

[3] C. Lim and A. Nirmalathas and M. Bakaul and P. Gamage and K. L. Lee and Y. Yang and D. Novak and R. Waterhouse, "Fiber-Wireless Networks and Subsystem Technologies," Journal of Lightwave Technology, vol. 28, no. 4, pp. 390-405, Feb 2010.

[4] A. de la Oliva, J. A. Hernandez, D. Larrabeiti and A. Azcorra, "An overview of the CPRI specification and its application to C-RAN-based LTE scenarios," IEEE Communications Magazine, vol. 54, no. 2, pp. 152-159, February 2016.

[5] P. T. Dat, A. Kanno, N. Yamamoto and T. Kawanishi, "5G transport networks: the need for new technologies and standards," IEEE Communications Magazine, vol. 54, no. 9, pp. 18-26, September 2016.

[6] A. Delmade, D. Venkitesh and R. D. Koilpillai, "Performance comparison of optical fronthauling techniques for centralized radio access network," IEEE ANTS Conference, November 2016.

[7] http://www.3gpp.org/technologies/keywords-acronyms/98-lte

[8] Peter Thornycroft, "Gigabit Wi-Fi, 802.11ac in depth," AIRHEADS Conference, ARUBA Networks, March 2013.

[9] B. Farhang-Boroujeny and H. Moradi, "OFDM Inspired Waveforms for 5G," IEEE Communications Surveys \& Tutorials, vol. 18, no. 4, pp. 2474 2492, Fourthquarter 2016.

[10] S. Cho, H. S. Chung, C. Han, S. Lee, and J. H. Lee, "Experimental Demonstrations of Next Generation Cost-Effective Mobile Fronthaul with IFoF technique," Optical Fiber Communication Conference and Exhibition (OFC), paper M2J.5, OFC 2015. 
[11] D. Wake, A. Nkansah, N. J. Gomes, G. de Valicourt, R. Brenot, M. Violas, Z. Liu, F. Ferreira, and S. Pato, "A Comparison of Radio Over Fiber Link Types for the Support of Wideband Radio Channels," Journal of Lightwave Technology, vol. 28, no. 16, pp. 2416-2422, August, 2010.

[12] M. Xu, J. Zhang, F. Lu, Y. Wang, D. Guidotti and G. K. Chang, "Investigation of FBMC in mobile fronthaul networks for 5G wireless with timefrequency modulation adaptation," 2016 Optical Fiber Communications Conference and Exhibition (OFC), paper W3C.2, pp. 1-3, March 2016.

[13] J. Zhang, M. Xu, J. Wang, F. Lu, L. Cheng, M. Zhu, I. Khalil, J. Yu and G. K. Chang, "Carrier aggregation for MMW inter-RAT and intraRAT in next generation heterogeneous mobile data network based on optical domain band mapping," 2015 European Conference on Optical Communication (ECOC), pp. 1-3, September 2015.

[14] Colm Browning1, Arman Farhang, Arsalan Saljoghei, Nicola Marchetti, Vidak Vujicic, Linda E. Doyle and Liam P. Barry, "5G Wireless and Wired Convergence in a Passive Optical Network using UF-OFDM and GFDM,", IEEE International Conference on Communications - International workshop on the main trends in 5G networks (MT5Gnet), May 2017.

[15] "Huawei and NTT DoCoMo reach $11 \mathrm{Gbps}$ speeds in 5G Japanese field trial," available at http://www.zdnet.com/article/ huawei-and-ntt-docomo-reach-11gbps-speeds-in-5g-japanese-field-trial/.

[16] A. Aminjavaheri, A. Farhang, A. Rezazadeh Reyhani and B. FarhangBoroujeny, "Impact of timing and frequency offsets on multicarrier waveform candidates for 5G," Signal Processing and Signal Processing Education Workshop (SP/SPE), 2015 IEEE, pp. 178-183, 2015.

[17] N. Michailow, M. Matth, I. S. Gaspar, A. N. Caldevilla, L. L. Mendes, A. Festag and G. Fettweis, "Generalized Frequency Division Multiplexing for 5th Generation Cellular Networks," IEEE Transactions on Communications, vol. 62, no. 9, pp. 3045-3061, September 2014.

[18] A. Farhang, N. Marchetti and L. E. Doyle, "Low-Complexity Modem Design for GFDM," IEEE Transactions on Signal Processing, vol. 64, no. 6, pp. 1507-1518, March 2016.

[19] V. Vakilian, T. Wild, F. Schaich, S. ten Brink and J. F. Frigon, "Universal-filtered multi-carrier technique for wireless systems beyond LTE,” 2013 IEEE Globecom Workshops (GC Workshops), pp. 223-228, December 2013.

[20] C. Herbert, D. Jones, A. Kaszubowska-Anandarajah, B. Kelly, M. Rensing, J. O'Carroll, R. Phelan, P. Anandarajah, P. Perry, L. p. Barry and J. O'Gorman, "Discrete mode lasers for communication applications," IET Optoelectronics, vol. 3, no. 1, pp. 1-17, February 2009.

[21] Nokia Siemens Networks, "Introducing LTE with maximum reuse of GSM assets," white paper, 2011, http://www.gsma.com/spectrum/ wpcontent/uploads/2012/03/lte1800mhzwhitepaper0.9.pdf.

[22] 4G America Community, "5G Spectrum Recommendation," White Paper, October 2015. http://www.5gamericas.org/files/9114/9324/1786/ 5GA_5G_Spectrum_Recommendations_2017_FINAL.pdf.

[23] Robin Gerzaguet, Nikolaos Bartzoudis, Leonardo Gomes Baltar, Vincent Berg, Jean-Baptiste Doré, Dimitri Kténas, Oriol Font-Bach, Xavier Mestre, Miquel Payaró, Michael Färber and Kilian Roth, "The 5G candidate waveform race: a comparison of complexity and performance," EURASIP Journal on Wireless Communications and Networking, Vol. 2017, pages 13, January 2017.

[24] A. RezazadehReyhani and B. Farhang-Boroujeny, "Asynchronous Performance of Circularly Pulse-Shaped Waveforms for 5G," IEEE Global Communications Conference (GLOBECOM), December 2016. 